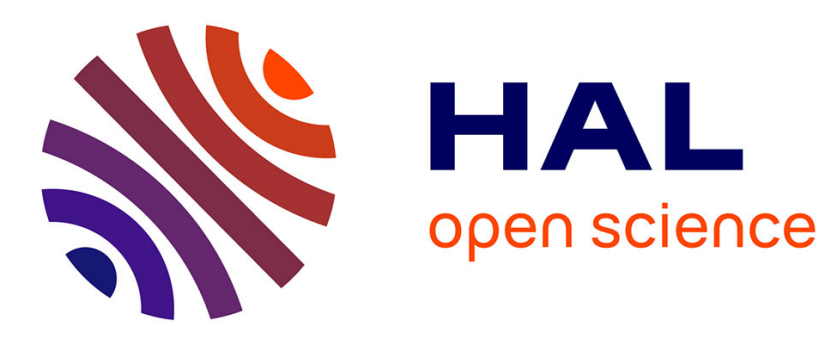

\title{
Les expériences françaises de réseaux de villes, des dynamiques pour de nouveaux territoires
}

Frédéric Tesson

\section{To cite this version:}

Frédéric Tesson. Les expériences françaises de réseaux de villes, des dynamiques pour de nouveaux territoires. Flux - Cahiers scientifiques internationaux Réseaux et territoires, 1997, 27-28, pp.25-39. halshs-00009025

\section{HAL Id: halshs-00009025 https://shs.hal.science/halshs-00009025}

Submitted on 12 Feb 2006

HAL is a multi-disciplinary open access archive for the deposit and dissemination of scientific research documents, whether they are published or not. The documents may come from teaching and research institutions in France or abroad, or from public or private research centers.
L'archive ouverte pluridisciplinaire HAL, est destinée au dépôt et à la diffusion de documents scientifiques de niveau recherche, publiés ou non, émanant des établissements d'enseignement et de recherche français ou étrangers, des laboratoires publics ou privés. 


\section{Frédéric Tesson}

Les expériences françaises de réseaux de villes, des dynamiques pour de nouveaux territoires

L'expression "réseau de villes" s'inscrit dans de multiples problématiques avec des acceptions très diverses, il est donc fondamental de préciser tout de suite le sens que nous lui donnons. "Réseau de villes" fait référence ici aux expériences françaises d'une politique d'aménagement et de développement, encouragée par la D.A.T.A.R. depuis la fin des années $80^{1}$, fondée sur la coopération volontaire de maires de villes intermédiaires dans la discontinuité spatiale. La notion de réseau est considérée ici comme un procès, un mode de fonctionnement d'un groupe social. Dans ce sens, si on préfère souvent à "réseau de villes" l'expression "alliance de villes", on voit qu'en ce qui concerne la relation qui s'instaure entre les maires il s'agit bien d'un réseau d'acteurs.

Notre objectif n'est pas ici de faire une présentation exhaustive et une analyse en profondeur de cette politique, ni même d'en décrire toutes les expériences ${ }^{2}$, mais plutôt de les mettre en perspective en considérant l'hypothèse selon laquelle les dynamiques territoriales sont largement déterminées par les interactions entre quatre formes d'organisations territorialisées: systèmes urbains, systèmes productifs, réseaux de transport et réseaux de communications. Trois questions fondamentales issues de cette hypothèse émergent nécessairement lorsqu'on parle de "réseaux de villes" en ces termes :

- les expériences de réseaux de villes peuvent-elles être considérées comme une ou des dynamiques territoriales?

- si tel est le cas, dans quelle mesure l'hypothèse proposée ici est-elle supportée empiriquement ? Et par là, quels sont les rapports entre les réseaux de villes et les formes d'organisations territorialisées précitées?

- à travers ce prisme, et de façon transversale, comment se traduisent les questions d'efficacité et de justice?

Dans un premier temps, nous montrerons comment les réseaux de villes, en entraînant les élus locaux dans un nouveau rapport, non seulement à l'espace, mais aussi au territoire et à ses règles, constituent bien une forme de dynamique territoriale induisant des évolutions en termes d'efficacité et de justice, cette dernière prenant tout son sens ici à

\footnotetext{
${ }^{1}$ Sans nier qu'il existe d'autres coopérations, nous nous appuyons ici sur les réseaux de villes répertoriés par la DATAR puisque c'était là le corpus de notre thèse.

${ }^{2}$ Pour cela se reporter aux références bibliographiques.
} 
travers l'équité territoriale contenue dans la notion d'aménagement du territoire. Ce regard nous amènera également à expliciter le sens dans lequel nous faisons référence à cette notion de réseau.

Dans un second temps, en nous appuyant à titre d'exemple sur les systèmes urbains et le système politico-administratif, nous montrerons que les réseaux de villes sont largement déterminés par les diverses formes d'organisations territorialisées par rapport auxquelles ils doivent se positionner clairement. Le champ des réseaux de villes, en faisant explicitement référence à l'ensemble de ces formes, nous permet de bien comprendre les interactions qui se développent entre elles. On voit bien ici qu'il s'agit d'une dynamique territoriale qui se positionne par rapport à chacune de ces formes d'organisation territorialisées, de leur évolution et de leurs multiples interactions.

C'est à travers une lecture transversale à ces deux axes que nous tenterons de comprendre en quels termes se posent les questions d'efficacité et de justice, sans toutefois proposer une réponse qui nous fuit encore.

\section{1- Les réseaux de villes : une dynamique territoriale}

Lorsqu'on parle de réseau on a souvent du mal à voir le territoire, l'un et l'autre ayant plutôt tendance à être opposés, le réseau étant souvent présenté comme niant le territoire. L'ouvrage "réseau et territoire, significations croisées", publié aux éditions de l'Aube en 1997 et reprenant les travaux de l'atelier "réseaux et territoires" du GDR "réseaux", montre bien qu'il y existe des connexions très fortes, les réseaux servant de nouveaux territoires. Notre travail de recherche développé dans une thèse intitulée : "les réseaux de villes en France, recherche sur le rapport de l'élu local à l'espace" 3 montre la complémentarité de ces deux concepts pour un groupe social bien spécifique : les élus locaux. La dimension du concept de réseau que nous avons animée dans cette recherche s'écarte un peu des réseaux techniques pour s'appuyer sur l'organisation en réseau, le mode de fonctionnement. Nous nous appliquerons, dans un premier point à expliciter ce que nous entendons par mode de fonctionnement en réseau, pour montrer ensuite comment cette figure singulière représentée par les réseaux de villes dans leur manifestation concrète peut être considérée comme une dynamique largement territoriale.

\section{1- Pour une dimension organisationnelle du concept de réseau, ou le réseau comme mode de fonctionnement des individus et des groupes}

${ }^{3}$ Thèse de doctorat de géographie et aménagement, soutenue le 10 avril 1996 à Pau, directeur de recherche : X. Piolle. 
Pour légitimer l'emploi du terme réseau dans l'expression "réseau de villes", il faut chercher dans les fondements de l'analyse du lien social. En effet, en sociologie, "un réseau peut être défini, ... , comme une communauté potentielle d'action... Dans le réseau, ce qui unifie l'action est en permanence le fait de l'acteur et de sa participation volontaire, plus ou moins éphémère, non le fait d'un système qui structure ou organise l'action." (JUAN S., 1991). Cette analyse ouvre vers une autre dimension du comportement de l'individu, une autre logique, un autre mode de fonctionnement. Cette grille prend tout son sens avec la mobilité sociale actuelle, la complexification et le déploiement des relations individuelles sur un champ spatial plus vaste. Les cadres traditionnels construits dans une approche du territoire (famille, village, quartier) sont transgressés, les catégories (sociales, ethniques, spatiales...) sont bousculées, et ce mouvement est largement favorisé par la multiplication des moyens de communication, par l'accès de plus en plus facile aux réseaux techniques.

L'analyse des réseaux économiques, du mode de fonctionnement en réseau des chefs d'entreprises, nous éclaire également sur ce que recouvre cette dimensions et sur les caractéristiques qui la déclinent. C'est ainsi qu'émerge une réflexion sur le concept de réseau en tant que nouveau mode d'organisation. Le réseau en économie représenterait “des modes d'organisations hybrides qui s'inscrivent d'un point de vue théorique dans un dépassement de la dualité firme/marché." (LECOQ B., 1991). Il fonctionnerait sous forme de contrats externes, implicites, entre entreprises. Ses modèles sont multiples et le réseau, en économie, semble représenter un degré supérieur de complexité. Que ce soit dans le fonctionnement interne ou dans les relations externes, le réseau semble s'imposer comme une alternative à la firme. L'analyse du fonctionnement en réseau à l'intérieur même de l'entreprise, nous renseigne sur quelques unes des principales caractéristiques de ce mode de fonctionnement. Si on s'appuie sur C. NEUSCHWANDER (1991) et sur N. ALTER (1990) pour définir le mode de fonctionnement en réseau, on peut mettre en évidence ses caractéristiques. Ainsi le mode de fonctionnement en réseau c'est à la fois échange et solidarité, souplesse et solidité ainsi que dynamisme et efficacité, et ce, sans hiérarchie ni contrainte (N. ALTER). On peut aussi ajouter la relation de confiance et de partenariat contenue dans le réseau dont l'efficacité provient de la mise en commun de ressources (C. NEUSCHWANDER). Souplesse et flexibilité se traduisent par des expressions comme "flux tendus", “juste à temps", que l'on retrouve dans le langage des chefs d'entreprises. Ainsi, le développement s'inscrit dans la souplesse, une flexibilité telle, qu'elle s'oppose à la règle, et transgresse, par le réseau, les “territoires” organisés.

Mais cette transgression des territoires existants ne renie pas toute forme de dynamique territoriale. En effet, le réseau peut prendre des formes telles que les Systèmes Industriels Localisés par exemple, qui sont, en quelques sortes des recompositions territoriales souvent très fortes. On voit nettement poindre ici des considérations 
d'efficacité sans que l'esprit de justice, entendu ici comme un souci d'équité territoriale, ne soit, à aucun moment, une préoccupation affichée par les chefs d'entreprises. C'est là toute la spécificité des réseaux économiques qui tient aussi pour les réseaux sociaux.

C'est ce contexte qui nous a poussé à aller voir du côté des élus locaux si un mode de fonctionnement analogue était repérable. Notre hypothèse initiale, fondée sur le caractère extrêmement territorial de cette sphère, complètement liée à des espaces parfaitement bornés imposant leurs frontières à la légitimité de l'action des élus, nous faisait pencher pour une incompatibilité majeure d'un détachement du territoire. Pourtant, lorsqu'est apparue la politique des réseaux de villes, et surtout ses premières expériences concrètes, il nous a semblé qu'il s'agissait là des prémices de l'accès, pour les élus locaux, à ce type de comportement réticulaire. En effet, dans notre thèse nous montrons à cet égard que les réseaux de villes, du moins dans les discours qu'ils produisent ${ }^{4}$, répondent aux caractéristiques de ce mode de fonctionnement. Par la coopération qu'ils induisent, par le discours volontariste qu'ils affichent, par le projet qu'ils mettent sans cesse en avant, par la souplesse qu'ils adoptent et les partenariats qu'ils mettent en place, les élus locaux lancés dans ces expériences semblent faire évoluer leur mode de fonctionnement vers le réseau. Il faut noter que, comme pour l'individu qui reste attaché à toutes les formes du fonctionnement territorial (quartier, village, famille), ou l'entreprise qui ne renie pas, en dépit de l'évolution, les avantages et le fonctionnement de la firme, l'élu local ne remplace pas le fonctionnement induit par le territoire par une logique réticulaire, ces deux modes de fonctionnement se complètent et se renforcent dans le souci d'une efficacité accrue sur tous les plans de son évaluation. En effet, si l'avenir de ses concitoyens passe par une ouverture à la logique réticulaire, sa réélection, qui est sans doute le projet le plus fort de ce type d'acteur, passe par une action au plus près de son territoire, action qui possède ses canons relevant de la logique induite par des règles très strictes et éminemment territoriale.

\section{2- Des réseaux de villes pour un nouveau territoire}

Si les réseaux de villes sont souvent comparés à des alliances de maires, nous pensons qu'ils se distinguent de l'étiquette de "club" qui leur est souvent attribuée. Considérer le réseau de villes comme un Club, revient à nier totalement l'importance du territoire, or force est de constater qu'il est encore bien présent dans quasiment l'ensemble des expériences. Ce phénomène est à mettre en relation avec une question que se posent la plupart des chercheurs et des acteurs étrangers aux réseaux de villes : pourquoi, dans un

\footnotetext{
${ }^{4}$ Nous avons conscience qu'il ne s'agit pas ici d'une petite nuance, mais force est de constater que le discours, en ce qui concerne cette sphère singulière des élus locaux, n'est pas neutre, induit des prises de consciences et révèlent par là une première évolution.
} 
contexte de mondialisation des échanges et de globalisation de l'économie et des marchés, Belfort, par exemple, pour aborder certaines préoccupations, échanger sur certaines expériences, ne chercherait pas s'associer avec Pau ou Niort plutôt qu'avec Colmar et St Louis ? Autrement dit la proximité spatiale est-elle une garantie de proximité des problèmes ? Le Club des villes TGV, par exemple, traduit bien cette préoccupation d'associer les villes qui connaissent les même types de problèmes. Et il me semble bien que cette question est la bonne, un Club est bien une réponse appropriée pour remédier à ce besoin d'échange légitime entre des villes ayant les mêmes problèmes.

Mais c'est aussi pour ces mêmes raisons que les réseaux de villes ne sont pas des clubs. En effet, ils s'appuient sur une question territoriale forte, un territoire qui n'est pas forcément celui, politico-administratif, qui s'impose à ces villes, mais un territoire tout de même, presque un système à l'intérieur duquel tout ce qu'il advient de positif ou de négatif à des répercussions du même ordre, une "onde de choc", pour toutes ses composantes pour reprendre la définition d'A. PRED (1977). Au sein d'un club c'est différent, les effets produits sur la ville d'Annecy par le TGV ne sont pas les mêmes que ceux rencontrés par la ville de Poitiers. Si l'échange d'expériences est fructueux, les problèmes d'une des villes n'entraînent pas de difficultés majeures, directement induites, pour les autres.

De fait, les réseaux de villes entraînent souvent une conscience territoriale forte (tableau $\mathbf{n}^{\circ} \mathbf{1}$ ) et développent des relations qui, s'il elles ne relèvent pas de la proximité contiguë, se réfèrent à un "non-éloignement" pour reprendre une formule de X. Piolle. Certains en viennent même à intégrer complètement l'interstice en devenant des S.I.V.O.M. ou en élargissant leurs partenariats de façon significative aux acteurs du milieu environnant, c'est le cas du Triangle ou de Oise la Vallée. On voit bien ici que les expériences de réseaux de villes témoignent d'une nouvelle définition, d'une recomposition des territoires. Situés principalement sur des marges territoriales (européenne, nationale ou régionale), ou en marge des grands pôles de développement, nous y reviendrons, la volonté consiste en une recomposition territoriale plus pertinente, plus fonctionnelle. Il n'est pas question, à ma connaissance, de revendiquer un redécoupage, de partir à la recherche de l'optimum dimensionnel, dont L. ORTIZ (1994) a montré qu'il s'agissait d'un mythe, il s'agit simplement de gagner en cohérence aux marges des territoires politico-administratifs, en arrêtant de postuler qu'ils correspondent au seul périmètre possible de réponse à l'ensemble des problèmes posés ${ }^{5}$.

Les réseaux de villes sont donc bien des dynamiques territoriales nouvelles se positionnant sur de nouveaux territoires ou sur des territoires différents de ceux que

\footnotetext{
${ }^{5}$ Voir à ce sujet le positionnement du maire de Pau face à la volonté de M. Inchauspé de créer, par amendement à la loi d'orientation pour l'aménagement et le développement du territoire du 4 février 1995, une région Adour et un département Basque.
} 
l'institution a découpé. Ce sera là l'objet de notre seconde partie.

\section{3- Les réseaux de villes et l'aménagement du territoire : l'équité en question}

Fonctionner en réseau pour des élus locaux modifie à la fois leur rapport à l'espace et au territoire mais aussi leur rapport à la règle induite par l'organisation territoriale. Cette règle qui attribue aux élus des compétences propres issues des lois sur la décentralisation et qui fait tomber dans l'escarcelle de la région la compétence d'aménager le territoire. Par l'intermédiaire des réseaux de villes, les maires trouvent un outil qui leur permet de prendre des positions et de faire émerger des projets relevant de cette compétence qu'ils n'ont pas. Ainsi, ils prennent position sur les grands schémas de la discussion desquels ils sont exclus (routiers et autoroutiers, ferroviaires, hospitaliers, universitaires,...) et par là modifient les données du problème en pesant de tout leur poids sur les décisions.

Cette question soulève le problème de la justice ou plutôt du couple équité/légitimité qui, selon nous, traduit le mieux cette notion dans le cas qui nous intéresse. En effet, pour ces maires de villes intermédiaires, se lancer dans un réseau de villes, signifie œuvrer pour l'aménagement du territoire. En animant la fibre aménagiste les maires concernés se placent résolument dans une perspective de justice territoriale, de redistribution de la richesse, pour contrer la logique de concentration des investissements et des projets dans les métropoles dont le rapport Guichard de 1986 préconisait le renforcement.

Il est certain que cette lutte pour le développement, qui se structure hors du système institutionnel classique et de ses règles, place les réseaux de villes face à d'autres lectures de la justice. En effet, cette équité au nom d'un aménagement du territoire s'appuie sur une volonté des élus locaux, hors qu'advient-il des territoires sur lesquels il n'existe pas de projets volontaristes de cet ordre ? Cette prime à l'engagement est-elle compatible avec la notion d'équité et quelle justice offre-t-elle réellement ?

Dans le même ordre d'idée, les élus agissent dans le cadre de ces expériences sans reconnaissance électorale et sur un territoire illégitime. Et même s'ils peuvent objecter, et ils ne s'en privent pas, que l'intercommunalité classique n'est pas beaucoup plus démocratique, la question du choix des projets à favoriser, aussi consensuel qu'ils puissent être, et ils sont le plus souvent choisis pour le consensus qu'ils engendrent, ne manque pas de soulever le problème de la justice démocratique de l'action des maires dans ce cadre.

Persuadés d'œuvrer pour l'intérêt général et celui de leurs concitoyens, les maires des villes en réseau se retrouvent ainsi accusés "d'antidémocratisme", leurs revendications taxées d'injustices. Il semble donc qu'il y ait deux niveaux de justice en cause dans les réseaux de villes. Le premier, dans la dimension d'aménagement du territoire que les élus accordent à cette politique qui place ces expériences dans un jeu 
d'équilibre entre les métropoles et les villes intermédiaire à un échelon national ou régional, et qui, s'il s'appuie sur le volontariat des maires ne souffre pas de contradiction majeure ${ }^{6}$. Le second, dans l'aspect non démocratique des décisions, prises au niveau des élus sur des questions et des territoires illégitimes, qui, à ce niveau-là, peut poser question.

Nous ne cherchons pas ici à donner de réponse à la question de la justice en matière de réseau de villes, nous voulons simplement formuler les questions telles qu'elles se posent dans ce cadre, à savoir : Un aménagement du territoire fondé sur le volontarisme du local est-il équitable? Quelle est la légitimité des maires d'une commune-centre lorsqu'il décident d'œuvrer pour le développement d'un territoire plus large ?

Pour conclure ce premier point on peut affirmer que les réseaux de villes, lus à travers le prisme scientifique du concept de réseau considéré dans sa dimension organisationnelle, constituent bien des dynamiques territoriales originales et non, comme on le souligne parfois, des clubs de villes partageant des expériences et des problèmes spécifiques ${ }^{7}$. De plus, il faut souligner que ces expériences soulèvent le problème qui correspond à la thématique de ce séminaire concernant les notions d'efficacité et de justice. Placés dans une problématique d'aménagement du territoire les maires des villes en réseau œuvrent pour une équité plus grande dans la répartition des richesses entre les métropoles et les villes intermédiaires, mais fonctionnant en réseau dans un objectif d'efficacité supérieure, ils ne détiennent finalement aucune légitimité démocratique pour ce faire.

\section{2- Les réseaux de villes : les figures de l'organisation territoriale en question}

Il nous semble particulièrement intéressant, dans ce second point, de lire les expériences des réseaux de villes à travers les formes d'organisations territoriales évoquées dans les axes problématiques. En effet, les réseaux de villes se positionnent clairement par rapport à l'ensemble de ces organisations :

- par rapport aux systèmes urbains, puisque les réseaux de villes se situent dans une perspective de remise en cause de la hiérarchie (armature urbaine) et comme une alternative multipolaire à la métropolisation annoncée comme irrémédiable. Dans cette catégorie nous plaçons également le système politico-administratif, qui peut, selon nous,

\footnotetext{
${ }^{6}$ Sauf à se demander ce qu'attendent réellement Lyon et Lille qui toutes deux engagent une procédure de ce type, qui, selon nous, n'est pas adaptée à leur qualité de métropole, voire est antinomique vis à vis de l'esprit même de la politique tant si on regarde les expériences déjà engagées que si on fouille un peu dans les discours de l'Etat.

${ }^{7}$ Loin d'être contradictoire, l'existence du Club des réseaux de villes montre bien qu'un réseau de villes est un objet à part entière, ayant sa logique et sa dynamique territoriale. Cette entité existant, elle pose problème et nécessite échange et transfert d'innovation sur ce thème, le Club représente l'outil de cet échange.
} 
être également considéré comme une forme d'organisation territorialisée par rapport à laquelle les réseaux de villes doivent également se positionner. En effet, ces alliances sont héritées de la décentralisation et de l'accroissement des pouvoirs locaux et bousculent quelque peu l'emboîtement classique des territoires politico-administratifs.

- vis-à-vis des systèmes productifs, dans la mesure où les expériences étudiées représentent clairement une volonté de repositionnement sur le marché national et mondial des sites dans la perspective de la globalisation croissante des échanges. Dans le même sens, les réseaux sociaux ne peuvent pas être écartés. Leur analyse et le constat de la mobilité accrue des individus - dont on voit bien par ailleurs le rapport avec l'évolution du système productif et les conséquences sur les systèmes urbains et les réseaux de transport et de communications - sont déterminants pour comprendre la dynamique territoriale qui nous intéresse ici.

- relativement aux réseaux de transport, qui sont une des préoccupations majeures des réseaux de villes, que ce soit dans le but d'améliorer les liaisons entre les villes associées et de favoriser ainsi la mobilité interne, pour assurer une meilleure desserte depuis l'extérieur voire parfois afin d'optimiser l'utilisation des infrastructures existantes. Les réseaux de communications sont aussi au cœur de cette dynamique territoriale résolument moderniste. Il s'agit ici d'un positionnement vis à vis des réseaux techniques dans leur ensemble.

A titre d'exemple nous porterons uniquement ici un regard sur le positionnement des réseaux de villes par rapport aux organisations institutionnelles du territoire, mais cette lecture semble pouvoir s'accorder aux autres organisations territoriales, notamment aux systèmes de villes, mais aussi aux réseaux économiques et techniques.

- Réseaux de villes et organisation institutionnelle du territoire : la quête d'une cohérence

En géographie le concept de système urbain est fondé sur la vision du système selon A. PRED (1977) : "un ensemble national ou régional de villes qui sont interdépendantes dans le sens où chaque changement significatif dans l'activité économique de l'une des villes du système, produira directement ou indirectement quelques modifications sur les autres." (PUMAIN, D. 1992). Cette approche des relations intercités met en avant l'échelon géographique régional comme niveau de repérage du système et il semble en effet que ce dernier dépasse le local pour se retrouver à une échelle "méso". Mais au-delà de l'approche scientifique, la hiérarchie de l'armature urbaine et les liens qu'elle entretient avec l'organisation institutionnelle du territoire, constituent des référents incontournables lorsqu'on approche les expériences de "réseaux de villes". Sans que l'on puisse parler de 
remise en cause, c'est bien dans l'optique d'un positionnement par rapport à un découpage territorial (départemental et régional) qui conditionnerait l'ensemble des politiques et contraindrait les projets dans ses frontières que les maires de ces quelques soixante communes françaises ont décidé de choisir l'alliance à distance pour organiser le développement. Le regard que nous portons ici tend, non pas à dresser une typologie qui scinderait en multiples catégories bien distinctes l'objet "réseau de villes", mais plutôt à proposer une unité qui fait ressortir des grandes constantes traduisant le positionnement de ces maires vis-à-vis de l'organisation institutionnelle du territoire.

* Le réseau de villes, un outil pour des villes intermédiaires...

Si on regarde la situation démographique des communes lancées dans des expériences de réseaux de villes ${ }^{8}$, nous relevons tout de suite une grande hétérogénéité (tableau $\mathbf{n}^{\circ}$ 2). Il est difficile, en effet, de penser qu'un réseau de villes qui implique la commune de Mende (11 286 habitants) et un autre bâti avec Nantes (244 995 habitants), aient les mêmes ambitions. En outre, ni l'une ni l'autre ne sont réellement des exceptions; certes, 41 communes sur 63 (soit 65\%) comptent moins de 60000 habitants, mais force est de constater qu'à l'intérieur de ce groupe la progression est régulière. En effet, quasiment toutes les tailles sont représentées : 8 cités ont moins de 20000 habitants, 10 entre 20 et 30000,5 entre 30 et 40000,10 entre 40 et 50000 et 8 entre 50 et 60000 . La même analyse peut être faite au niveau des unités urbaines même si en fait l'engagement des maires ne concerne que les commune-centre hormis quelques districts dont on sait qu'il ne sont pas représentatif de la réalité urbaine. Il n'existe donc pas une unité démographique, pas même de seuil d'apparition des réseaux de villes.

$\mathrm{Si}$ on se penche maintenant sur les situation administratives (tableau $\mathbf{n}^{\circ} \mathbf{3}$ ), qui tendent à traduire la place des villes dans la hiérarchie régionale, on remarque que la multiplicité des situations semble incompatible à tout rapprochement. Entre un simple chef-lieu de canton comme Aix les Bains et une métropole régionale telle que Rennes en passant par des sous-préfectures (St Dizier par exemple), des chefs lieux de départements (Pau ou Alençon) et des préfectures de région (Poitiers ou Caen), le poids et les préoccupations sont très différents.

Cependant, en restant sur le plan démographique et administratif, si toutes ces villes sont différentes, il existe un invariant majeur : "l'intermédiarité". En effet, elles sont toutes en marge des grands seuils démographiques dont le franchissement entraîne des plus-values considérables pour la visibilité et le développement.

Considérons tout d'abord l'échelle européenne. Nantes compte, intra-muros, près de 245000 habitants; de fait, elle entre dans la catégorie des communes de plus de 200000 habitants, celles qui comptent sur le territoire français. Si on considère l'agglomération

\footnotetext{
${ }^{8}$ Sans nier qu'il existe d'autres coopérations, nous nous appuyons ici sur les réseaux de villes répertoriés par la DATAR puisque c'était là le corpus de notre thèse.
} 
toute entière, échelon indispensable pour comparer les villes au sein de l'Europe, Nantes est la 8ème unité urbaine française, juste en deçà de la barre des 500000 habitants ${ }^{9}$. De ce fait, elle n'est pas considérée comme capable de s'imposer en tant que métropole internationale dans la future Europe des villes qui se dessine. La situation est plus critique encore pour les neuf autres villes en réseau enserrées dans des agglomérations de plus de 200000 habitants ${ }^{10}$.

Ensuite, dans le même ordre d'idée, de "petites" préfectures régionales, sans statut métropolitain (Poitiers, Caen, Châlons sur Marne...), ou de "gros" chefs-lieux départementaux (Pau, Nîmes...) connaissent des problèmes de lisibilité à un niveau plus national, même s'ils regardent aussi vers l'Europe. Enfin, quelques-unes de ces villes (Pau, Chambéry...) mais également de plus petites, sans statut administratif particulier (Saintes, Lorient...) ou dans des "coins de France" très reculés (Mende, Chalon sur Saône...), luttent avant toute chose pour une reconnaissance régionale ${ }^{11}$ face à des métropoles jugées "castratrices".

Europe, France, région, la principale différence entre toutes ces villes intermédiaires se situe donc sur le plan de l'échelle de référence et donc du seuil en deçà duquel les élus classent leur cité. Mais les considérations scalaires : lisibilité européenne, nationale ou régionale, n'altèrent pas le type fondé sur la position intermédiaire des villes et leur incapacité à atteindre seules les objectifs de développement qu'elles se fixent. La coopération des élus doit permettre, à l'ensemble et à chaque ville, de franchir un seuil, de déclencher une dynamique de développement supérieure à celle atteinte par la seule action quotidienne territoriale et à celle envisageable par de nouvelles actions isolées.

Par l'intermédiarité des villes concernées par ces expériences on voit que l'outil réseau de villes se place incontestablement dans une logique de remise en cause du "tout métropolitain" et par là des évolutions de l'organisation territoriale du pays présentées comme inéluctables.

* ... et sur les marges géographiques et administratives

Au-delà de "l'intermédiarité", pour montrer l'unité et l'homogénéité du type "réseau de villes", et pour illustrer son positionnement face aux grandes organisations territoriales, on peut ajouter à cette analyse la position périphérique de ces villes. Celle-ci se décline en trois grandes catégories de référents. Leur situation géographique indique que les acteurs qui se sont saisis de cet outil se situent tous sur les marges. Marges géographiques ou marge du développement, la grille de lecture classique

\footnotetext{
${ }^{9}$ Classement des unités urbaines françaises selon l'I.N.S.E.E. Nantes compte 496000 habitants en 1990.

${ }^{10}$ Ce seuil quantitatif est celui retenu par l'I.N.S.E.E. pour réunir, derrière Paris, les plus grandes unités urbaines françaises.

${ }^{11}$ Certaines alliances, fondées sur une forte proximité, revendiquent un bassin démographique, une autre forme de territoire, pour appuyer leur lisibilité. C'est ainsi que le Triangle (Bar le Duc, Vitry le François, St Dizier) se présente comme un bassin de 200000 habitants (!) alors que la somme des trois agglomérations ne dépasse pas le seuil des 100000 .
} 
“centre/périphérie" semble pertinente pour observer cet impact spatial. Les réseaux de villes se développent principalement :

- sur les marges du territoire français,

- sur les marges régionales,

- en périphérie des grands pôles de développement.

Le schéma de localisation des réseaux de villes (Schéma $\mathbf{n}^{\circ} \mathbf{1}$ ), dont le fond fait apparaître les limites administratives régionales, s'avère être un bon révélateur de ce phénomène. Il met en évidence sept réseaux de villes dans le grand Ouest français ${ }^{12}$, deux autres dans l'Est de la France ${ }^{13}$, et un dernier tout à fait au Nord ${ }^{14}$. Ainsi onze réseaux de villes sur dix neuf, soit 35 villes sur les 63 engagées sont situés aux confins du territoire. Dès lors, l'association de ces villes peut se lire dans une logique très pragmatique, s'affirmer dans un espace français très centralisé.

Ce type de marginalité n'est pas le seul; d'autres réseaux, parfois les mêmes, associent des villes au-delà des frontières régionales ${ }^{15}$. De plus, sur les huit réseaux transgressant ces limites, sept concernent des villes situées aux confins de leurs régions respectives ${ }^{16}$, sur "les marches régionales" pour reprendre l'expression de J. L. CHAUZY ${ }^{17}$. Cette expression porte en elle la volonté des acteurs de réaliser, à travers ces villes, un autre usage de la frontière, espace de contact et d'échanges, de concurrence et de richesse; ces villes sont pionnières, en route vers une "nouvelle frontière". La "marche" se révèle particulièrement pertinente pour traduire, mieux que la marge, la dynamique qui habite les acteurs des réseaux de villes. Son auteur la justifie en montrant que les villes situées sur ces périphéries régionales, pour subsister face à la métropole de la région ou tout simplement pour gagner en cohérence au-delà des limites institutionnelles, ne peuvent se contenter de regarder le territoire politico-administratif auquel elles appartiennent. Ainsi, loin de penser que ces associations nuisent à la cohérence du territoire, il milite pour que les acteurs considèrent cette position marginale comme favorable au dépassement des frontières. Cette transgression ne doit donc pas être ressentie comme une agression, au contraire, elle constitue une chance (peut-être la seule ?) pour les villes concernées, mais aussi pour la région, de s'ancrer dans le territoire national et d'entamer avec les régions

\footnotetext{
${ }^{12}$ Normandie Métropole (Caen-Rouen-Le Havre), Tours-Alençon-Le Mans, Nantes-Brest-Angers-RennesLe Mans, St Malo-St Brieuc-Vannes-Quimper-Lorient, AIRE 198 (Poitiers-Niort-Angoulême-La Rochelle), Cognac Océan (Cognac-Rochefort-Saintes) et Pyrénées Métropole (Pau-Tarbes-Lourdes). Soit 25 villes.

13 Rhin Sud (Colmar-Mulhouse-Belfort-Montbéliard-Héricourt-St Louis) et Annecy-ChambéryAnnemasse-Aix les Bains. Soit 10 villes.

${ }^{14}$ Nord Littoral (Calais-Boulogne sur Mer-Dunkerque). Soit 3 villes.

15 Cf. tableau ${ }^{\circ} 1$

${ }^{16}$ Parmi ceux-ci le réseau des grandes villes de l'Ouest, bien qu'interrégional, n'entre pas dans ce type particulier de marginalité.

${ }^{17}$ Président du Conseil Economique et Social de Midi Pyrénées (discours à la première rencontre des acteurs de réseaux de villes organisée à Poitiers en juillet 1993).
} 
voisines des coopérations bénéfiques à tous. Cette approche est très riche et peut s'appliquer à d'autres réseaux qui, sans être interrégionaux, recèlent dans leur constitution les traits d'une cohérence à la périphérie du territoire régional. C'est le cas notamment de Côte d'Opale, aux confins de la région Nord-Pas de Calais, qui s'affirme comme un trait d'union indispensable à celle-ci, avec la Grande Bretagne via le tunnel sous la Manche. L'exemple de Oise la Vallée, à l'articulation de la Picardie et de l'Ile de France, plus proche de Paris que d'Amiens (sa préfecture régionale), ou de Beauvais (siège de son conseil général), est aussi révélateur du rôle que le réseau peut et compte jouer au sein de sa région et de son département. Annecy-Chambéry-Annemasse-Aix les Bains, dans un même esprit, donne un rôle majeur au sillon alpin en relation avec Genève, contrebalançant et complétant l'armature urbaine d'une région déjà largement multipolaire avec Lyon, St Etienne et Grenoble. Enfin, le cas de Cognac Océan, sur les "marches" des deux Charentes, peut être lu de cette façon à l'échelon départemental.

Nous voyons donc que la position périphérique de ces réseaux les positionnent dans une problématique claire d'aménagement du territoire avec un souci de compléter, par la multipolarité, une armature urbaine riche en ville moyenne mais pauvre en réelles métropoles, et ce par delà les logiques des découpages politico-administratifs classiques. De ce fait les réseaux de villes sont bien des réponses, sans juger de leur pertinence ni de leur efficacité, à une forme d'organisation territoriale, celle des institutions.

Une autre forme de marginalité géographique apparaît à travers la situation des réseaux par rapport aux grands pôles de développement européens. L'Ile de France fait incontestablement partie de ceux-ci et cette région est encerclée par une couronne de huit réseaux de villes, au sein du Bassin Parisien ${ }^{18}$. De même, trois réseaux ${ }^{19}$ se développent en marge de la "Banane bleue" européenne, autre pôle de développement majeur, mis en évidence par les chorèmes de R. BRUNET. Cette position dénote une même volonté : réagir face aux grandes observations du monde portées par les universitaires et les propectivistes, mais aussi "accrocher le bon wagon", celui du développement.

Cette entrée par la marge, au-delà du schéma, permet de mettre en évidence deux derniers types de marginalité plus isolés. Le premier concerne PIB'SUD (Châlon sur Saône-Communauté urbaine Creusot-Montceau); ces deux villes sont en marge du développement, marquées par des problèmes majeurs de restructuration économique dont le réseau de villes est l'héritier. Enfin, la dernière figure est représentée par Estelle (RodezAurillac-Mende), trois villes situées à la charnière entre l'urbain et le rural. Ces alliances s'inscrivent dans l'urgence, celle de répondre à une attente très forte de la part de citoyens isolés sur les plans géographique, social et économique.

${ }^{18}$ Normandie Métropole, Oise la Vallée, Reims-Troyes-Châlons sur Marne-Epernay, Troyes-AuxerreSens-Chaumont, le Triangle, Orléans-Blois-Tours, Tours-Alençon-Le Mans etChateauroux-IssoudunVierzon-Bourges. Soit 25 villes.

${ }^{19}$ Nord Littoral, Rhin Sud et Annecy-Chambéry-Annemasse-Aix les Bains. Soit 13 villes. 
Cette lecture des réseaux de villes français ne nous révèle pas toute leur réalité, ce n'était pas l'objectif, mais elle montre qu'il s'agit bien là d'un outil pour se repositionner à une autre échelle par rapport aux grands ensembles régionaux et à leurs frontières politico-administratives. Pour compléter la hiérarchie imposée par le système productif et le système politico-administratif, les réseaux de villes prônent une alliance et une complémentarité territoriale d'un autre type. Il est difficile pour autant, du fait de leur dimension éminemment politique, de mettre en parallèle les réseaux de villes et les analyses actuelles des systèmes urbains tels que les montrent des auteurs comme F. Damette, D. Pumain ou Th. Saint Julien. En effet, le caractère volontariste de ces alliances s'accommode mal des analyses universitaires et géographiques sur les relations interurbaines même si, lorsqu' on regarde les cartes produites dans ce cadre on voit apparaître certains espaces vides, dans lesquels se développent des réseaux de villes. Pour aller un peu plus loin, et reprendre une réflexion de C. Rozenblat, on pourrait dire que les systèmes urbains tels que les met en évidence l'analyse spatiale, recenseraient les réseaux de villes potentiels. Par là, la géographie peut être considérée comme une discipline efficace d'aide à la décision. Reste à savoir l'usage qu'en feront les acteurs qui décident en dernier recours de travailler ensemble.

\section{Pour conclure ce second point}

Nous avons volontairement insisté ici sur une des organisations territorialisées : le système institutionnel. Cela se justifie par le fait que les réseaux de villes sont initiés par des élus pour lesquels cette organisation prime naturellement sur tous les autres, du fait de leur représentativité, de leurs compétences et de leurs devoirs. Mais il semble qu'une telle lecture, positionnant les réseaux de villes vis à vis d'autres systèmes d'organisation, soit possible, notamment par rapport aux systèmes productifs et aux réseaux techniques.

On peut souligner pour finir que cette lecture ne manque pas de questionner les notions de justice et d'équité. Si l'esprit va dans le sens d'un meilleur équilibre entre les métropoles et les villes moyennes, qu'advient-t-il des espaces ruraux interstitiels ? Ces expériences peuvent-elles imposer une loi volontariste qui favoriserait uniquement les espaces capables, par leurs acteurs, de se mobiliser ? Et aujourd'hui, la mise en réseau de certaines métropoles ne constitue-t-elle pas une fuite en avant de ce type d'expérience ?

\section{Conclusion}

Les expériences concrètes de réseaux de villes montrent qu'il faut nécessairement dépasser une première lecture qui peut faire apparaître cet outil comme une alliance de villes sans grande référence territoriale, à l'image de certains clubs qui émergent depuis quelques années en France. L'effet de non-éloignement repéré dans ces réseaux et les 
évolutions vers des figures plus continues, prenant en compte l'interstice et pensant œuvrer, par l'intermédiaire des villes, pour le développement global d'un territoire bien plus vaste et à géométrie variable, font de cet outil une réelle dynamique territoriale. Cet dynamique s'appuie sur l'animation d'un réseau d'acteurs, fondé par les élus locaux, dont le mode de fonctionnement, dicté par le projet de développement, dépasse la hiérarchie pour s'imprégner de souplesse et d'horizontalité.

Dans ce mouvement, clairement présenté comme l'émanation d'une quête d'efficacité pour le développement, nous avons tenté de présenter en quels termes se pose la question de la justice. Selon nous, celle-ci est à rattacher à la notion d'équité véhiculée par l'aménagement du territoire duquel émane cette politique. De ce point de vue, les réseaux de villes soulèvent deux questions fondamentales qui restent entières :

- Dans quelle mesure un aménagement volontariste peut-il être équitable alors qu'il exclut tous les espace sur lesquels il n'est pas formulé de projet?

- Comment un maire de commune-centre peut-il revendiquer le droit d'œuvrer pour le développement d'un ensemble territorial sur lequel il n'a aucune compétence ni légitimité ?

Dynamique pour un territoire pas nécessairement calqué sur ceux que l'institution a défini, le réseau de villes doit se positionner par rapport à diverses figures d'organisations territorialisées. Nous avons favorisé ici une lecture des réseaux de villes dans leur rapport à l'organisation politico-administrative du territoire. Outil animé par des acteurs situés sur les marges géographiques et institutionnelles, les réseaux de villes questionnent la cohérence des frontières sans remettre en question leur nécessité mais en s'opposant à leur imperméabilité. Dit autrement, l'outil réseau de villes, tel qu'il est animé aujourd'hui par les acteurs qui l'utilisent, remet en question un postulat très jacobin qui veut que le département, la région, voire la France, sont des systèmes à l'intérieur desquels doit se passer le développement. Les réseaux de villes proposent des solutions pour les marges de ces entités plus administratives que géographiques pour une meilleure cohérence de leur développement.

Ici aussi intervient le problème de la justice, très lié à celui de l'efficacité. En effet, la volonté des acteurs entrant dans un réseau est-elle la garantie d'une pertinence de l'alliance ? Dans ce sens, des études d'analyse spatiale, telles que les développent certains géographes, semble pouvoir aider à la décision, mais la volonté de travailler ensemble pour des acteurs, surtout politique, ne se décrète pas et répond à des critères que le scientifique ne fait pas forcément entrer dans son analyse.

\section{BIBLIOGRAPHIE}

- ALTER Norbert, 1990, La gestion du désordre en entreprise, Paris : L'Harmattan, 207 p.

BALLIGAND Jean Pierre, MAQUART Daniel, 1990, La fin du territoire jacobin, Paris : Albin Michel, $221 \mathrm{p}$. 
- BECKOUCHE Pierre, SAVY Michel, VELTZ Pierre, 1988, "Nouvelle économie, nouveaux territoires", Supplément à la Lettre de la DATAR, n³, juin, 4 p.

- CHERREQUE Jacques, 1990, "Déclaration du gouvernement dans le grand débat sur l'aménagement du territoire à l'assemblée nationale", le 29 Mai, 11 p.

- D.A.T.A.R., 1990, "Réseaux de villes et aires métropolitaines", dossier DATAR, la Lettre de la DATAR, mars, 4 p.

- D.A.T.A.R., 1991, En Europe, des villes en réseaux, Paris : La documentation française, coll. informations et analyses, $109 \mathrm{p}$.

- DERYCKE Pierre Henri, 1993, “Théorie des réseaux de villes”, in les villes, lieux d'Europe, dir. A. SALLEZ, Paris : DATAR-Ed.de l'Aube, p.95-105.

- DUPUY Gabriel, 1991, L'urbanisme des réseaux, Paris : Armand Collin, 198 p.

- FABRE Jean.(rapporteur pour le Conseil Economique et Social), 1991, "le développement des villes françaises de dimension européenne et les réseaux de villes", Journal officiel, avis et rapports du Conseil Economique et Social, n5, 18 mars, p.98-114.

- GUIGOU Jean Louis, 1990, "réseaux de villes", Urbanisme, n²33, décembre 1989 - janvier 90, p.62-63.

- JUAN Salvador, 1991, Sociologie des genres de vie, morphologie culturelle et dynamique des positions sociales, Paris : PUF, coll. Le sociologue, 384 p.

- LECOQ Bruno, 1991, “Organisation industrielle, organisation territoriale : une approche intégrée fondée sur le concept de réseau", Revue d'économie régionale et urbaine, n³/4, p.321343.

- MAQUART Daniel, 1992, "Réseaux et territoires du politique”, entretien pour Flux, n8, avriljuin, p.40-45.

- NEUSCHWANDER Claude, 1995, Développement local et réseaux, entretien pour Flux, n²0, Avril-Juin, p. 46-49.

- NEUSCHWANDER Claude et VERDIE M., 1990, Etude sur les réseaux de villes, Paris : groupe TEN, $103 \mathrm{p}$.

- OFFNER Jean Marc, 1992, "Sociologie des réseaux, morphogénèse des réseaux techniques", convention romande de troisième cycle de sociologie, première session, Yverdon les 27 et 28 novembre.

- ORTIZ Laure, 1994, “Espace et efficacité de l'action, le mythe de l'optimum dimensionnel”, in le renouveau de l'aménagement du territoire en France et en Europe, dir. J. C. NEMERY, Paris : Economica, p.183-200.

- PIOLLE Xavier, SOUBEYRAN Olivier, 1991, "Réseaux de villes, régions, eurorégions", rapport à la DATAR, le 8 juillet, 2 p.

- PRED Alan, 1977, City systems in advanded economies, Londres : Hutchinson.

- PUMAIN Denise et OFFNER Jean Marc (dir.), 1996, Réseaux et territoires, significations croisées, G.D.R. "réseaux", Paris : Editions de l'Aube, 1996, 288 p.

- PUMAIN Denise, 1992, "Les systèmes de villes", chapitre 34 de l'Encyclopédie de géographie, dir. A.BAILLY, R.FERRAS, D.PUMAIN, Paris, Economica, p.645-663.

- ROCARD Michel, 1991, Chartes d'objectifs et réseaux de villes, circulaire n³.678/SG aux préfets de région, le 17 avril, 8 p.

- ROYOUX Dominique, 1995, "Réseaux de villes et politiques publiques locales", 
communication au colloque "villes moyennes, espace, société, patrimoine”, Mâcon, 26 et 27 janvier, $14 \mathrm{p}$.

- ROYOUX Dominique, 1993, "Réseaux, territoire, dynamiques locales : stratégies, questions de recherche, valorisation - Valorisation mode d'emploi -", Métropolis, n98/99: chercheurs en ville, p.77-81

- ROYOUX Dominique, 1991, "La démarche des villes moyennes en Poitou-Charente", communication pour la journée d'étude de l'INUDEL : réseaux de villes en région Rhône-Alpes, quels contenus, quelles stratégies ?, 17 décembre, p.27-39.

- ROYOUX Dominique, TESSON Frédéric, THOVISTE Laurent, 1995, Les réseaux de villes à l'aube de la loi sur le développement et l'aménagement du territoire, document de synthèse pour la DATAR.

- St JULIEN Thérèse, 1992, "Définition : réseau, armature, système urbain, glissement de sens, nouvelles questions, ré-écriture ?", l’Information géographique, n56, p.63-70.

- TESSON Frédéric, 1997, "Pour un partenariat au service du projet : les réseaux de villes", in Le Maire en trepreneur, actes du colloque : Le maire entrepreneur?, Pau : Presses Universitaire de Pau, pp.485-493.

- TESSON Frédéric, 1997, "Du pays au réseau de villes, le mode de fonctionnement comme lien", in Pays, réseaux et territoires en projet, J. P. Jambes dir., Pau : Hégoa n 19, 1996, pp. 7384.

- TESSON Frédéric, 1995, "Fiches de présentation des réseaux de villes", dossier réalisé pour le colloque de St Dizier, les 3 et 4 février, 17 p.

- TESSON Frédéric et GUERIT Frank, 1996, "Discours politique ou renouveau territorial, le réseau de villes Pau-Tarbes-Lourdes", in Les territoires du quotidien, G. Di Méo dir., Caen : L'Harmattan, pp. 163-184.

- TESSON Frédéric et PIOLLE Xavier, 1995, Le réseau de villes, objet scientifique? rapport scientifique sur commande du Plan Urbain, juin 1995, 80 p.

- TESSON Frédéric, ROYOUX Dominique et THOVISTE Laurent, 1996, Les réseaux de villes, Voiron : La Lettre du cadre territorial, coll. Dossiers d'expert, 1996, 165 p. 



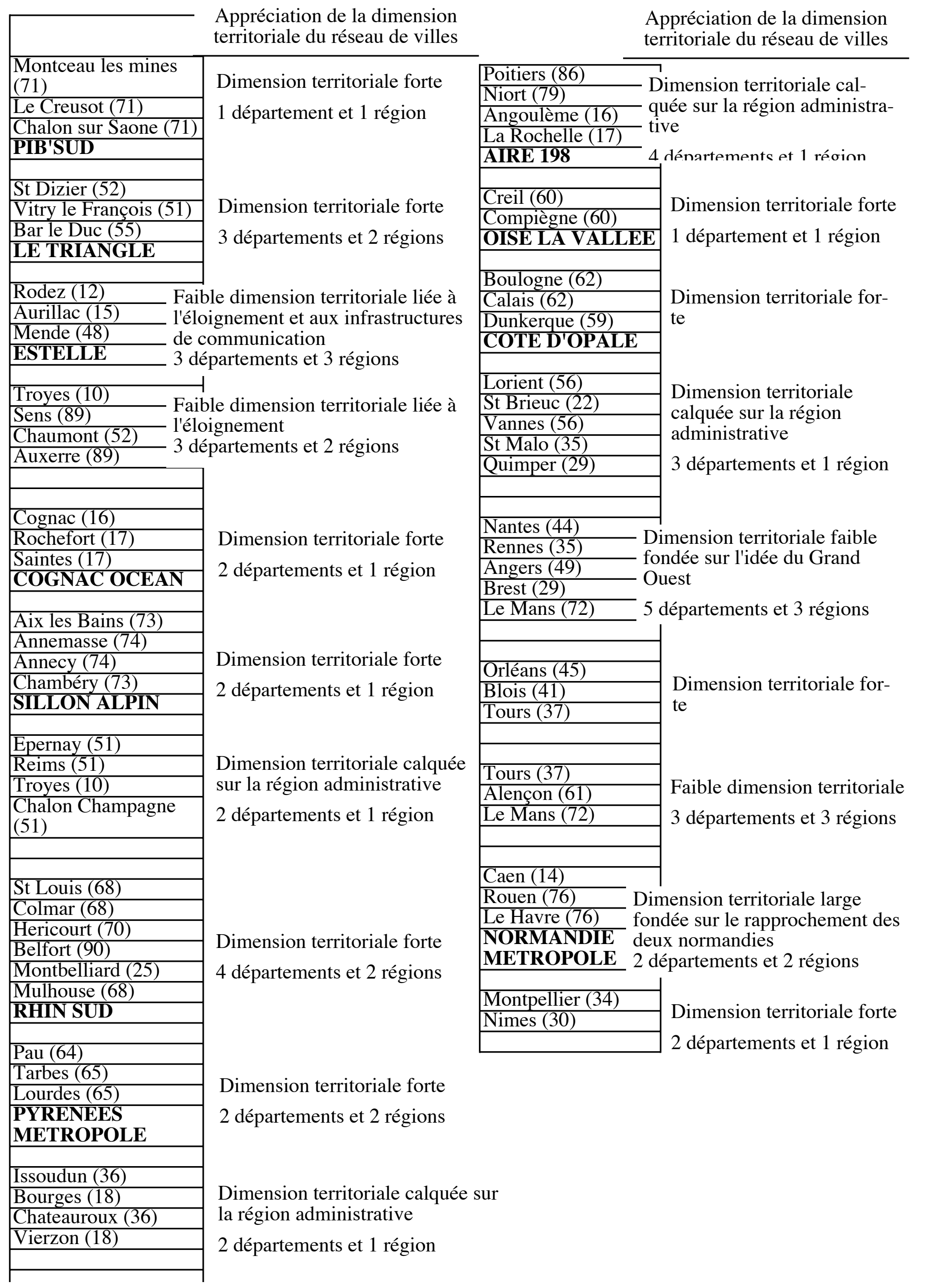


Tableau $\mathrm{n}^{\circ} 2$ : La population concernée par les réseaux de villes

\begin{tabular}{|c|c|c|}
\hline & $\begin{array}{l}\text { population } \\
\text { commune }\end{array}$ & $\begin{array}{c}\text { population } \\
\text { unité } \\
\text { urbaine }\end{array}$ \\
\hline Montceau les mines (71) & 22959 & 47283 \\
\hline Le Creusot (71) & 28909 & 40903 \\
\hline Chalon sur Saone (71) & 54575 & 77764 \\
\hline PIB'SUD & 106443 & 165950 \\
\hline St Dizier (52) & 33552 & 40097 \\
\hline Vitry le François (51) & 17030 & 19919 \\
\hline Bar le Duc (55) & 17545 & 21388 \\
\hline LE TRIANGLE & 68127 & 81404 \\
\hline Rodez (12) & 24701 & 39017 \\
\hline Aurillac (15) & 30773 & 36069 \\
\hline Mende (48) & 11286 & 11286 \\
\hline ESTELLE & 66760 & 86372 \\
\hline Troyes (10) & 59255 & 122763 \\
\hline Sens (89) & 27082 & 36221 \\
\hline Chaumont (52) & 27041 & 27988 \\
\hline Auxerre (89) & 38819 & 42005 \\
\hline & 152197 & 228977 \\
\hline$\overline{C o g n a c ~(16)}$ & 19528 & 27468 \\
\hline Rochefort (17) & 25561 & 35598 \\
\hline Saintes (17) & 25874 & 27003 \\
\hline COGNAC OCEAN & 70963 & 90069 \\
\hline Aix les Bains (73) & 24683 & 35472 \\
\hline Annemasse (74) & 27669 & 98758 \\
\hline Annecy (74) & 49644 & 126729 \\
\hline Chambéry (73) & 54120 & 103283 \\
\hline SILLON ALPIN & 156116 & 364242 \\
\hline Epernay (51) & 26682 & 34038 \\
\hline Reims (51) & 180620 & 206437 \\
\hline Troyes (10) & 59255 & 122763 \\
\hline Chalon Champagne (51) & 48423 & 61452 \\
\hline & 314980 & 424690 \\
\hline St Louis (68) & 19547 & 33509 \\
\hline Colmar (68) & 63498 & 83816 \\
\hline Hericourt (70) & 9742 & 9742 \\
\hline Belfort (90) & 50125 & 77844 \\
\hline Montbelliard (25) & 29005 & 117510 \\
\hline Mulhouse (68) & 108357 & 223856 \\
\hline RHIN SUD & 280274 & 546277 \\
\hline Pau (64) & 82157 & 144674 \\
\hline Tarbes (65) & 47566 & 77787 \\
\hline Lourdes (65) & 16300 & 16300 \\
\hline PYRENEES & 146023 & 238761 \\
\hline Iscoudun (36) & 13859 & 13850 \\
\hline Issoudun (36) & 13859 & 13859 \\
\hline Bourges (18) & 75609 & 94731 \\
\hline Chateauroux (36) & 50969 & 67090 \\
\hline Vierzon (18) & 32235 & 36049 \\
\hline & 172672 & 211729 \\
\hline
\end{tabular}

\begin{tabular}{|c|c|c|}
\hline Poitiers (86) & 78894 & 107625 \\
\hline Niort (79) & 57012 & 66792 \\
\hline Angoulème (16) & 42876 & 102908 \\
\hline La Rochelle (17) & 71094 & 100264 \\
\hline AIRE 198 & 249876 & 377589 \\
\hline Creil (60) & 31956 & $\overline{97119}$ \\
\hline Compiègne (60) & 41896 & 67057 \\
\hline OISE LA VALLEE & 73852 & 164176 \\
\hline Boulogne (62) & 43678 & 91249 \\
\hline Calais (62) & 75309 & 101768 \\
\hline Dunkerque (59) & 70331 & 190879 \\
\hline COTE D'OPALE & 189318 & 383896 \\
\hline \begin{tabular}{|l} 
Lorient (56) \\
\end{tabular} & 59271 & 115488 \\
\hline St Brieuc (22) & 44752 & 83861 \\
\hline Vannes (56) & 45644 & 45644 \\
\hline St Malo (35) & 48057 & 48057 \\
\hline \multirow{2}{*}{ Quimper (29) } & 59427 & 65954 \\
\hline & 257151 & 359004 \\
\hline \begin{tabular}{|l} 
Nantes (44) \\
\end{tabular} & 244995 & 496078 \\
\hline Rennes (35) & 197536 & 245065 \\
\hline Angers (49) & 141404 & 208282 \\
\hline Brest (29) & 147956 & 201480 \\
\hline \multirow[t]{2}{*}{ Le Mans (72) } & 145502 & 189107 \\
\hline & 877393 & 1340012 \\
\hline Orléans (45) & 105111 & 243153 \\
\hline Blois (41) & 49318 & 65132 \\
\hline \multirow[t]{2}{*}{ Tours (37) } & 129509 & 282152 \\
\hline & 283938 & 590437 \\
\hline \begin{tabular}{|l} 
Tours (37) \\
\end{tabular} & 129509 & 282152 \\
\hline Alençon (61) & 29988 & 42471 \\
\hline \multirow[t]{2}{*}{ Le Mans (72) } & 145502 & 189107 \\
\hline & 304999 & 513730 \\
\hline \begin{tabular}{|l} 
Caen (14) \\
\end{tabular} & 112856 & 191490 \\
\hline Rouen (76) & 102723 & 380161 \\
\hline Le Havre (76) & 195854 & 253627 \\
\hline \multirow{2}{*}{$\begin{array}{l}\text { NORMANDIE } \\
\text { METROPOLE }\end{array}$} & & \\
\hline & 411433 & 825 \\
\hline \multirow{3}{*}{$\begin{array}{l}\text { Montpellier (34) } \\
\text { Nimes (30) }\end{array}$} & 207996 & 248303 \\
\hline & 128471 & 138527 \\
\hline & 336467 & 386830 \\
\hline
\end{tabular}

Remarques :

En gras : nom et population de l'ensemble du réseau de villes.

(51) : département dans lequel est située la commune 


\begin{tabular}{|c|c|}
\hline & $\begin{array}{l}\text { position administrative } \\
\text { des villes en réseau }\end{array}$ \\
\hline Montceau les mines (71) & ch.lieu de canton \\
\hline Le Creusot (71) & ch.lieu de canton \\
\hline Chalon sur Saone (71) & ch.lieu d'arrondissement \\
\hline \multicolumn{2}{|l|}{ PIB'SUD } \\
\hline St Dizier (52) & ch.lieu d'arrondissement \\
\hline Vitry le François (51) & ch.lieu d'arrondissement \\
\hline Bar le Duc (55) & ch.lieu de département \\
\hline \multicolumn{2}{|l|}{ LE TRIANGLE } \\
\hline Rodez (12) & ch.lieu de département \\
\hline Aurillac (15) & ch.lieu de département \\
\hline Mende (48) & ch.lieu de département \\
\hline \multicolumn{2}{|l|}{ ESTELLE } \\
\hline Troyes $(10)$ & ch.lieu de département \\
\hline Sens (89) & ch.lieu d'arrondissement \\
\hline Chaumont (52) & ch.lieu de département \\
\hline Auxerre (89) & ch.lieu de département \\
\hline Cognac (16) & ch.lieu d'arrondissement \\
\hline Rochefort (17) & ch.lieu d'arrondissement \\
\hline Saintes (17) & ch.lieu d'arrondissement \\
\hline \multicolumn{2}{|l|}{ COGNAC OCEAN } \\
\hline Aix les Bains (73) & ch.lieu de canton \\
\hline Annemasse (74) & ch.lieu de canton \\
\hline Annecy (74) & ch.lieu de département \\
\hline Chambéry (73) & ch.lieu de département \\
\hline \multicolumn{2}{|l|}{ SILLON ALPIN } \\
\hline Epernay (51) & ch.lieu d'arrondissement \\
\hline Reims (51) & ch.lieu d'arrondissement \\
\hline Troyes (10) & ch.lieu de département \\
\hline Chalon Champagne (51) & ch.lieu de département \\
\hline St Louis (68) & 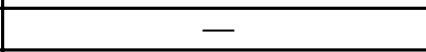 \\
\hline Colmar (68) & ch.lieu de département \\
\hline Hericourt (70) & ch.lieu de canton \\
\hline Belfort (90) & ch.lieu de département \\
\hline Montbelliard (25) & ch.lieu d'arrondissement \\
\hline Mulhouse (68) & ch.lieu d'arrondissement \\
\hline \multicolumn{2}{|l|}{ RHIN SUD } \\
\hline Pau (64) & ch.lieu de département \\
\hline Tarbes (65) & ch.lieu de département \\
\hline Lourdes (65) & ch.lieu de canton \\
\hline \multicolumn{2}{|l|}{$\begin{array}{l}\text { PYRENEES } \\
\text { METROPOLE }\end{array}$} \\
\hline Issoudun (36) & ch.lieu d'arrondissement \\
\hline Bourges (18) & ch.lieu de département \\
\hline Chateauroux (36) & ch.lieu de département \\
\hline Vierzon (18) & ch.lieu d'arrondissement \\
\hline
\end{tabular}

\begin{tabular}{|l|l|}
\hline Poitiers (86) & capitale régionale \\
\hline Niort (79) & ch.lieu de département \\
\hline Angoulème (16) & ch.lieu de département \\
\hline La Rochelle (17) & ch.lieu de département \\
\hline AIRE 198 & \\
\hline & \\
\hline Creil (60) & - \\
\hline Compiègne (60) & ch.lieu d'arrondissement \\
\hline OISE LA VALLEE & \\
\hline & \\
\hline Boulogne (62) & ch.lieu d'arrondissement \\
\hline Calais (62) & ch.lieu d'arrondissement \\
\hline Dunkerque (59) & ch.lieu d'arrondissement \\
\hline COTE D'OPALE & \\
\hline & \\
\hline Lorient (56) & ch.lieu d'arrondissement \\
\hline St Brieuc (22) & ch.lieu de département \\
\hline Vannes (56) & ch.lieu de département \\
\hline St Malo (35) & ch.lieu d'arrondissement \\
\hline Quimper (29) & ch.lieu de département \\
\hline & \\
\hline Nantes (44) & capitale régionale \\
\hline Rennes (35) & capitale régionale \\
\hline Angers (49) & ch.lieu de département \\
\hline Brest (29) & ch.lieu d'arrondissement \\
\hline Le Mans (72) & ch.lieu de département \\
\hline & \\
\hline Orléans (45) & capitale régionale \\
\hline Blois (41) & ch.lieu de département \\
\hline Tours (37) & ch.lieu de département \\
\hline & \\
\hline Tours (37) & ch.lieu de département \\
\hline Alençon (61) & ch.lieu de département \\
\hline Le Mans (72) & ch.lieu de département \\
\hline & \\
\hline Caen (14) & capitale régionale \\
\hline Rouen (76) & capitale régionale \\
\hline Le Havre (76) & ch.lieu d'arrondissement \\
\hline $\begin{array}{l}\text { NORMANDIE } \\
\text { METROPOLE }\end{array}$ & \\
\hline & ch.lieu de département \\
\hline Montpellier (34) & \\
\hline Nimes (30) & \\
\hline & \\
\hline &
\end{tabular}

